PROCEEDINGS OF THE

AMERICAN MATHEMATICAL SOCIETY

Volume 135, Number 3, March 2007, Pages 865-871

S 0002-9939(06)08592-3

Article electronically published on September 11, 2006

\title{
WHY IS ISOTROPY SO PREVALENT IN SPATIAL STATISTICS?
}

\author{
CHUNSHENG MA
}

(Communicated by Richard A. Davis)

\begin{abstract}
There are many reasons for the popular use of the isotropic or geometrically anisotropic covariance function and variogram in spatial statistics. A less known reason demonstrated in this paper is that an isotropic or geometrically anisotropic model would be the only choice in certain circumstances, for instance, when the underlying random field is smooth enough.
\end{abstract}

\section{INTRODUCTION}

A real-valued random field $\left\{Z(\mathbf{x}), \mathbf{x} \in \mathbb{R}^{d}\right\}$ is a family of real-valued random variables whose index set is $\mathbb{R}^{d}$. For modelling spatial data, a commonly required assumption on the dependence structure of the underlying random field is weak or second-order stationarity, which means that the expectation $\mathrm{E} Z(\mathrm{x})$ is a constant, and the covariance

$$
C(\mathbf{x})=\mathrm{E}\left[\left\{Z\left(\mathbf{x}+\mathbf{x}_{0}\right)-\mathrm{E} Z\left(\mathbf{x}+\mathbf{x}_{0}\right)\right\}\left\{Z\left(\mathbf{x}_{0}\right)-\mathrm{E} Z\left(\mathbf{x}_{0}\right)\right\}\right], \mathbf{x} \in \mathbb{R}^{d},
$$

depends only on the spatial lag $\mathbf{x}$, where $\mathbf{x}_{0} \in \mathbb{R}^{d}$ is arbitrary. One of the routine procedures to render a random field stationary in spatial statistics is to take differences and work on the increment field. A random field $\left\{Z(\mathbf{x}), \mathbf{x} \in \mathbb{R}^{d}\right\}$ is then said to be intrinsically stationary (or to have stationary increments), if for every fixed $\mathbf{x}_{0} \in \mathbb{R}^{d}$, the increment field $\left\{Z\left(\mathbf{x}+\mathbf{x}_{0}\right)-Z(\mathbf{x}), \mathbf{x} \in \mathbb{R}^{d}\right\}$ is stationary, or equivalently, if $\mathrm{E}\left\{Z\left(\mathbf{x}+\mathbf{x}_{0}\right)-Z\left(\mathbf{x}_{0}\right)\right\}$ is a constant, and the variogram

$$
\gamma(\mathbf{x})=\frac{1}{2} \operatorname{var}\left\{Z\left(\mathbf{x}+\mathbf{x}_{0}\right)-Z\left(\mathbf{x}_{0}\right)\right\}, \mathbf{x} \in \mathbb{R}^{d},
$$

is a function of the spatial lag $\mathbf{x}$ only.

A covariance function $C(\mathbf{x}), \mathbf{x} \in \mathbb{R}^{d}$, is obviously symmetric (i.e., $C(-\mathbf{x})=C(\mathbf{x})$, $\mathbf{x} \in \mathbb{R}^{d}$ ) and positive definite in the sense that the inequality

$$
\sum_{i=1}^{n} \sum_{j=1}^{n} a_{i} a_{j} C\left(\mathbf{x}_{i}-\mathbf{x}_{j}\right) \geq 0
$$

holds for every $n \in \mathbb{N},\left\{\mathbf{x}_{1}, \ldots, \mathbf{x}_{n}\right\} \subset \mathbb{R}^{d}$ and $\left\{a_{1}, \ldots, a_{n}\right\} \subset \mathbb{R}$. Conversely, a symmetric and positive definite function on $\mathbb{R}^{d}$ can always be thought of as the covariance function of a zero-mean Gaussian random field, according to Kolmogorov's

Received by the editors November 1, 2003 and, in revised form, October 1, 2005.

2000 Mathematics Subject Classification. Primary 62M30, 60G10; Secondary 60G60, 43A35, $86 \mathrm{~A} 32$.

Key words and phrases. Covariance, geometrically anisotropic, isotropic, negative definite, norm, positive definite, variogram.

(C)2006 American Mathematical Society Reverts to public domain 28 years from publication 
existence theorem, although it might not be the covariance function for a nonGaussian random field. For example, the positive definite function $\exp \left(-\|\mathbf{x}\|^{2}\right), \mathbf{x} \in$ $\mathbb{R}^{d}$, cannot be a covariance function associated with any log-Gaussian random field [18, where $\|\mathbf{x}\|=\left(\sum_{k=1}^{d} x_{k}^{2}\right)^{1 / 2}, \mathbf{x} \in \mathbb{R}^{d}$, is the Euclidean norm.

A variogram $\gamma(\mathbf{x}), \mathbf{x} \in \mathbb{R}^{d}$, is symmetric, nonnegative with $\gamma(\mathbf{0})=0$, and negative definite in the sense that the inequality

$$
\sum_{i=1}^{n} \sum_{j=1}^{n} a_{i} a_{j} \gamma\left(\mathbf{x}_{i}-\mathbf{x}_{j}\right) \leq 0
$$

holds for every integer $n \geq 2$, arbitrary $\mathbf{x}_{k} \in \mathbb{R}^{d}$ and $a_{k} \in \mathbb{R}(k \in\{1, \ldots, n\})$ subject to $\sum_{k=1}^{n} a_{k}=0$. On the other hand, for a given symmetric and negative definite function $\gamma(\mathbf{x})$ on $\mathbb{R}^{d}$ with $\gamma(\mathbf{0})=0$, we can find a zero-mean Gaussian random field with the covariance of Schoenberg-Lévy type (9], 14]), $\gamma\left(\mathbf{x}_{1}\right)+\gamma\left(\mathbf{x}_{2}\right)-\gamma\left(\mathbf{x}_{1}-\mathbf{x}_{2}\right)$, $\mathbf{x}_{1} \in \mathbb{R}^{d}, \mathbf{x}_{2} \in \mathbb{R}^{d}$, whose variogram coincides with $\gamma(\mathbf{x})$. Hence, the variogram of a Gaussian random field is essentially characterized by its negative definiteness. The reader is referred to [2] and 3] for properties of positive definite and negative definite functions.

An isotropic or radial covariance function (variogram) on $\mathbb{R}^{d}$ is a function of the Euclidean norm $\|\mathbf{x}\|$ only, and a geometrically anisotropic covariance function (variogram) on $\mathbb{R}^{d}$ depends only on $\|A \mathbf{x}\|$, where $A$ is a $d \times d$ matrix. Such models have been frequently employed in modelling spatial or spatio-temporal data (e.g., [4, [6], 12], 13], 14], 15, [22], and [23]), for which there appear to be several reasons. First, the correlation between two locations simply depends on the usual Euclidean distance, and the covariance function and variogram are invariant under rotation. Second, an integral representation was well established for the continuous isotropic positive definite function [21. Third, it possesses many attractive properties so that various statistical modelling procedures can be applied. For instance, the deformation modelling approach 20] utilizes a deformation of the real geographical domain into a new coordinate system where isotropic spatial correlation structure is modeled. Another reason is described in Section 2, where Theorem 2 indicates that an isotropic or geometrically anisotropic model would be the only possibility in case the mean square derivative exists for a univariate margin of the underlying random field.

Section 3 describes how an isotropic variogram is associated with two nonisotropic covariance functions, one being stationary and the other being nonstationary.

\section{MAin REsults}

By a homogeneous function $\gamma(\mathbf{x})$ on $\mathbb{R}^{d}$, we mean it satisfies

$$
\gamma(\alpha \mathbf{x})=|\alpha| \gamma(\mathbf{x}), \text { for all } \mathbf{x} \in \mathbb{R}^{d}, \alpha \in \mathbb{R} .
$$

In order to derive our main results, we need the following lemma, which is also of interest by itself.

Lemma 1. Let $\gamma: \mathbb{R}^{d} \rightarrow[0, \infty)$ be a homogeneous function. If $\gamma^{2}(\mathbf{x})$ is a variogram on $\mathbb{R}^{d}$, then

(i) $\gamma(\mathbf{x})$ is a seminorm on $\mathbb{R}^{d}$; 
(ii) $\gamma^{2}(\mathbf{x}), \mathbf{x} \in \mathbb{R}^{d}$, is a quadratic form, in the sense that the following parallelogram law holds for all $\mathbf{x}_{1}, \mathbf{x}_{2} \in \mathbb{R}^{d}$ :

$$
\gamma^{2}\left(\mathbf{x}_{1}+\mathbf{x}_{2}\right)+\gamma^{2}\left(\mathbf{x}_{1}-\mathbf{x}_{2}\right)=2\left\{\gamma^{2}\left(\mathbf{x}_{1}\right)+\gamma^{2}\left(\mathbf{x}_{2}\right)\right\}
$$

and

(iii) there exists a $d \times d$ matrix $A$ such that

$$
\gamma(\mathbf{x})=\|A \mathbf{x}\|, \mathbf{x} \in \mathbb{R}^{d} .
$$

Proof. (i) Being a variogram on $\mathbb{R}^{d}, \gamma^{2}(\mathbf{x})$ is negative definite. Its square root $\gamma(\mathbf{x})$ is subadditive [2]; namely,

$$
\gamma\left(\mathbf{x}_{1}+\mathbf{x}_{2}\right) \leq \gamma\left(\mathbf{x}_{1}\right)+\gamma\left(\mathbf{x}_{2}\right), \mathbf{x}_{1}, \mathbf{x}_{2} \in \mathbb{R}^{d} .
$$

As a result, $\gamma(\mathbf{x})$ is a seminorm on $\mathbb{R}^{d}$.

(ii) Since $\gamma^{2}(\mathbf{x})$ is negative definite on $\mathbb{R}^{d}$, we particularly choose $n=4, \mathbf{x}_{3}=$ $-\mathbf{x}_{1}, \mathbf{x}_{4}=-\mathbf{x}_{2}$, and $a_{1}=a_{3}=1, a_{2}=a_{4}=-1$, and obtain

$$
\begin{aligned}
0 & \geq \frac{1}{2} \sum_{i=1}^{4} \sum_{j=1}^{4} a_{i} a_{j} \gamma^{2}\left(\mathbf{x}_{i}-\mathbf{x}_{j}\right) \\
& =-2 \gamma^{2}\left(\mathbf{x}_{1}+\mathbf{x}_{2}\right)-2 \gamma^{2}\left(\mathbf{x}_{1}-\mathbf{x}_{2}\right)+\gamma^{2}\left(2 \mathbf{x}_{1}\right)+\gamma^{2}\left(2 \mathbf{x}_{2}\right) \\
& =-2 \gamma^{2}\left(\mathbf{x}_{1}+\mathbf{x}_{2}\right)-2 \gamma^{2}\left(\mathbf{x}_{1}-\mathbf{x}_{2}\right)+4 \gamma^{2}\left(\mathbf{x}_{1}\right)+4 \gamma^{2}\left(\mathbf{x}_{2}\right),
\end{aligned}
$$

where the last equality follows from (2.1). Thus

$$
\gamma^{2}\left(\mathbf{x}_{1}+\mathbf{x}_{2}\right)+\gamma^{2}\left(\mathbf{x}_{1}-\mathbf{x}_{2}\right) \geq 2\left\{\gamma^{2}\left(\mathbf{x}_{1}\right)+\gamma^{2}\left(\mathbf{x}_{2}\right)\right\}, \mathbf{x}_{1}, \mathbf{x}_{2} \in \mathbb{R}^{d} .
$$

On the other hand, it follows from (2.1) and (2.3) that

$$
\begin{aligned}
& \gamma^{2}\left(\mathbf{x}_{1}+\mathbf{x}_{2}\right)+\gamma^{2}\left(\mathbf{x}_{1}-\mathbf{x}_{2}\right) \\
\leq & \frac{1}{2}\left[\gamma^{2}\left\{\left(\mathbf{x}_{1}+\mathbf{x}_{2}\right)+\left(\mathbf{x}_{1}-\mathbf{x}_{2}\right)\right\}+\gamma^{2}\left\{\left(\mathbf{x}_{1}+\mathbf{x}_{2}\right)-\left(\mathbf{x}_{1}-\mathbf{x}_{2}\right)\right\}\right] \\
= & \frac{1}{2}\left\{\gamma^{2}\left(2 \mathbf{x}_{1}\right)+\gamma^{2}\left(2 \mathbf{x}_{2}\right)\right\} \\
= & 2\left\{\gamma^{2}\left(\mathbf{x}_{1}\right)+\gamma^{2}\left(\mathbf{x}_{2}\right)\right\} .
\end{aligned}
$$

Combining this with (2.3) yields that $\gamma^{2}: \mathbb{R}^{d} \rightarrow[0, \infty)$ obeys the parallelogram law.

(iii) As a quadratic form on $\mathbb{R}^{d}, \gamma^{2}(\mathbf{x})$ is of the form $\mathbf{x}^{\prime} \Lambda \mathbf{x}, \mathbf{x} \in \mathbb{R}^{d}$, where $\Lambda$ is a symmetric matrix. Since it is nonnegative for all $\mathbf{x} \in \mathbb{R}^{d}$, there exists a matrix $A$ such that $\Lambda=A^{\prime} A$. In other words, $\gamma(\mathbf{x})=\left(\mathbf{x}^{\prime} \Lambda \mathbf{x}\right)^{1 / 2}=\|A \mathbf{x}\|, \mathbf{x} \in \mathbb{R}^{d}$.

Although every norm in the plane is a variogram ([8], [10]), its square is no longer a variogram, except for a quadratic form. For example, $\left(\left|x_{1}\right|^{p}+\left|x_{2}\right|^{p}\right)^{2 / p}$ and $\max \left(x_{1}^{2}, x_{2}^{2}\right)$ are not variograms on $\mathbb{R}^{2}$, where $p \geq 1$ and $p \neq 2$.

One of our key results is the following.

Theorem 2. Let $f: \mathbb{R} \rightarrow \mathbb{R}$ be an even, nonconstant, and twice differentiable function, and let $\gamma: \mathbb{R}^{d} \rightarrow[0, \infty)$ be a homogeneous function. If $f(\gamma(\mathbf{x}))$ is a covariance function on $\mathbb{R}^{d}$, then $\gamma(\mathbf{x})$ must be of the form (2.2).

Proof. As a part of the proof, let us get insight into the role played by the function $f(x)$ and the smoothness of the underlying random field. Except for the degenerate 
case where $\gamma(\mathbf{x}) \equiv 0$ for all $\mathbf{x} \in \mathbb{R}^{d}$, there is a spatial lag $\mathbf{x}_{0} \in \mathbb{R}^{d}$ such that $\gamma\left(\mathbf{x}_{0}\right)>0$. Since $f(\gamma(\mathbf{x}))$ is a covariance function on $\mathbb{R}^{d}$,

$$
f(x)=f(|x|)=f\left(\gamma\left(\frac{\mathbf{x}_{0}}{\gamma\left(\mathbf{x}_{0}\right)} x\right)\right), x \in \mathbb{R},
$$

is a covariance function on the real line. Moreover, it is twice differentiable there, which implies the existence of the mean square derivative of the univariate process $\left\{Z\left(\mathbf{x}_{1} x\right), x \in \mathbb{R}\right\}$, where $\mathbf{x}_{1} \in \mathbb{R}^{d}$ is an arbitrary but fixed location. Examples of such univariate processes include $\left\{Z\left(x_{1}, 0, \ldots, 0\right), x_{1} \in \mathbb{R}\right\}, \ldots,\left\{Z\left(0, \ldots, 0, x_{d}\right)\right.$, $\left.x_{d} \in \mathbb{R}\right\}$.

According to a theorem of Slutsky, Theorem 1.4 of [7], $f^{\prime}(0)=0$ and $-f^{\prime \prime}(x)$ is a covariance function on $\mathbb{R}$, which implies that $f^{\prime \prime}(0)<0$.

For every nonzero $\alpha, f(\gamma(\alpha \mathbf{x}))=f(\alpha \gamma(\mathbf{x}))$ is clearly a covariance function on $\mathbb{R}^{d}$, whose variogram is $f(0)-f(\alpha \gamma(\mathbf{x}))$. It implies that $\alpha^{-2}\{f(0)-f(\alpha \gamma(\mathbf{x}))\}$ is a variogram on $\mathbb{R}^{d}$. So is

$$
\lim _{\alpha \rightarrow 0} \frac{f(0)-f(\alpha \gamma(\mathbf{x}))}{\alpha^{2}}=\lim _{\alpha \rightarrow 0} \frac{-\gamma(\mathbf{x}) f^{\prime}(\alpha \gamma(\mathbf{x}))}{2 \alpha}=-\frac{f^{\prime \prime}(0)}{2} \gamma^{2}(\mathbf{x}),
$$

by L'Hôpital's rule. Consequently, $\gamma^{2}(\mathbf{x})$ is a variogram on $\mathbb{R}^{d}$, and the desired result follows from Lemma 1 .

Under a stronger condition that $\gamma(\mathbf{x})$ is a norm on $\mathbb{R}^{d}$, Proposition 1 of $[5$ ] is obtained as a corollary of Theorem 2 The conclusion actually can be drawn among all homogeneous functions, and it excludes many possible candidates of $\gamma(\mathbf{x})$ besides non-Euclidean norms.

To illustrate the use of Theorem 2, consider the even and twice differentiable function

$$
f(x)=\sin \left(\frac{\pi}{2} e^{-|x|}\right), x \in \mathbb{R} .
$$

For a nonnegative and homogeneous function $\gamma(\mathbf{x})$ on $\mathbb{R}^{d}$, if $f(\gamma(\mathbf{x}))$ is a covariance function on $\mathbb{R}^{d}$, then $\gamma(\mathbf{x})$ is necessarily of the form (2.2). It is shown in 11 that $f(\|\mathbf{x}\|)$ is indeed a covariance function on $\mathbb{R}^{d}$. So is $f(\|A \mathbf{x}\|), \mathbf{x} \in \mathbb{R}^{d}$, for a $d \times d$ matrix $A$.

As another application of Theorem 2, we investigate the random field extension of a continuous-time autoregressive process on the real line to the spatial domain $\mathbb{R}^{d}$, and introduce spatial autoregressive random fields over $\mathbb{R}^{d}$ as well as related spatio-temporal models [13.

In terms of Schoenberg's theorem (Theorem 2.2 of [2, p. 74), a function $\gamma$ : $\mathbb{R}^{d} \rightarrow[0, \infty)$ is a variogram if and only if $1-\exp \{-\alpha \gamma(\mathbf{x})\}, \mathbf{x} \in \mathbb{R}^{d}$, is a variogram on $\mathbb{R}^{d}$ for all nonnegative constants $\alpha$. The permissibility of a product of this kind of variogram is examined in the next theorem.

Theorem 3. Let $\gamma: \mathbb{R}^{d} \rightarrow[0, \infty)$ be a homogeneous function, and let $\alpha_{k}(k=1,2)$ be positive constants.

(i) A necessary and sufficient condition for the function

$$
\left[1-\exp \left\{-\alpha_{1} \gamma(\mathbf{x})\right\}\right]\left[1-\exp \left\{-\alpha_{2} \gamma(\mathbf{x})\right\}\right], \mathbf{x} \in \mathbb{R}^{d},
$$

to be a variogram on $\mathbb{R}^{d}$ is that $\gamma(\mathbf{x})$ is of the form (2.2). 
(ii) The function

$$
\exp \left\{-\alpha_{1} \gamma(\mathbf{x})\right\}+\exp \left\{-\alpha_{2} \gamma(\mathbf{x})\right\}-\exp \left\{-\left(\alpha_{1}+\alpha_{2}\right) \gamma(\mathbf{x})\right\}, \mathbf{x} \in \mathbb{R}^{d},
$$

is a covariance function on $\mathbb{R}^{d}$ if and only if $\gamma(\mathbf{x})$ takes the form (2.2) .

Proof. We are going to prove statement (ii), which is clearly equivalent to statement (i).

Consider the function

$$
f(x)=\exp \left(-\alpha_{1}|x|\right)+\exp \left(-\alpha_{2}|x|\right)-\exp \left\{-\left(\alpha_{1}+\alpha_{2}\right)|x|\right\}, x \in \mathbb{R},
$$

which is obviously even, and twice differentiable on $\mathbb{R}$ with

$f^{\prime \prime}(x)=\alpha_{1}^{2} \exp \left(-\alpha_{1}|x|\right)+\alpha_{2}^{2} \exp \left(-\alpha_{2}|x|\right)-\left(\alpha_{1}+\alpha_{2}\right)^{2} \exp \left\{-\left(\alpha_{1}+\alpha_{2}\right)|x|\right\}, x \in \mathbb{R}$.

By Lemma 1, a necessary condition for (2.5) to be a covariance function on $\mathbb{R}^{d}$ is that $\gamma(\mathbf{x})$ takes the form (2.2).

On the other hand, suppose that $\gamma(\mathbf{x})$ is of the form (2.2). We now show that (2.5) is a covariance function on $\mathbb{R}^{d}$. To this end, it suffices to work on the special case where $\gamma(\mathbf{x})=\|\mathbf{x}\|, \mathbf{x} \in \mathbb{R}^{d}$. By Bochner's theorem,

$$
1-\left\{1-\exp \left(-\alpha_{1}\|\mathbf{x}\|\right)\right\}\left\{1-\exp \left(-\alpha_{2}\|\mathbf{x}\|\right)\right\}
$$

is a covariance function on $\mathbb{R}^{d}$ if its Fourier transform is nonnegative. This is confirmed by

$$
\begin{aligned}
& \int_{\mathbb{R}^{d}}\left[1-\left\{1-\exp \left(-\alpha_{1}\|\mathbf{x}\|\right)\right\}\left\{1-\exp \left(-\alpha_{2}\|\mathbf{x}\|\right)\right\}\right] \exp \left(\imath \boldsymbol{\omega}^{\prime} \mathbf{x}\right) d \mathbf{x} \\
= & \int_{\mathbb{R}^{d}} \exp \left(-\alpha_{1}\|\mathbf{x}\|+\imath \boldsymbol{\omega}^{\prime} \mathbf{x}\right) d \mathbf{x}+\int_{\mathbb{R}^{d}} \exp \left(-\alpha_{2}\|\mathbf{x}\|+\imath \boldsymbol{\omega}^{\prime} \mathbf{x}\right) d \mathbf{x} \\
& -\int_{\mathbb{R}^{d}} \exp \left\{-\left(\alpha_{1}+\alpha_{2}\right)\|\mathbf{x}\|+\imath \boldsymbol{\omega}^{\prime} \mathbf{x}\right\} d \mathbf{x} \\
= & {\left[\alpha_{1}\left(\|\boldsymbol{\omega}\|^{2}+\alpha_{1}^{2}\right)^{-\frac{d+1}{2}}+\alpha_{2}\left(\|\boldsymbol{\omega}\|^{2}+\alpha_{2}^{2}\right)^{-\frac{d+1}{2}}\right.} \\
& \left.-\left(\alpha_{1}+\alpha_{2}\right)\left\{\|\boldsymbol{\omega}\|^{2}+\left(\alpha_{1}+\alpha_{2}\right)^{2}\right\}^{-\frac{d+1}{2}}\right] c_{0} \\
\geq & 0, \boldsymbol{\omega} \in \mathbb{R}^{d},
\end{aligned}
$$

where $c_{0}=2^{d} \pi^{(d-1) / 2} \Gamma((d+1) / 2)$.

\section{THE ISOTROPIC VARIOGRAM}

\section{AND RELATED NON-ISOTROPIC COVARIANCE FUNCTIONS}

In this section we explore the relationship among an isotropic variogram and two closely related non-isotropic covariance functions, one stationary and the other nonstationary.

Suppose that $\left\{Z(\mathbf{x}), \mathbf{x} \in \mathbb{R}^{d}\right\}$ is a random field whose covariance function is of Schoenberg-Lévy type (9, 14]),

$$
\operatorname{cov}\left(Z\left(\mathbf{x}_{1}\right), Z\left(\mathbf{x}_{2}\right)\right)=g\left(\left\|\mathbf{x}_{1}\right\|\right)+g\left(\left\|\mathbf{x}_{2}\right\|\right)-g\left(\left\|\mathbf{x}_{1}-\mathbf{x}_{2}\right\|\right), \mathbf{x}_{1}, \mathbf{x}_{2} \in \mathbb{R}^{d},
$$

where $g(x)$ is a nonnegative function on $[0, \infty)$ with $g(0)=0$. A well-known example is the fractional Brownian field, where $g(x)=|x|^{\alpha}, x \in[0, \infty)$, and $\alpha \in(0,2]$ is a constant (cf., [19]). 
The function $g(\|\mathbf{x}\|)$ in (3.1) is nothing but the variogram associated with the random field $\left\{Z(\mathbf{x}), \mathbf{x} \in \mathbb{R}^{d}\right\}$, since

$$
\begin{aligned}
\frac{1}{2} \operatorname{var}\left(Z\left(\mathbf{x}_{1}\right)-Z\left(\mathbf{x}_{2}\right)\right) & =\frac{1}{2}\left\{\operatorname{var}\left(Z\left(\mathbf{x}_{1}\right)\right)+\operatorname{var}\left(Z\left(\mathbf{x}_{2}\right)\right)-2 \operatorname{cov}\left(Z\left(\mathbf{x}_{1}\right), Z\left(\mathbf{x}_{2}\right)\right)\right\} \\
& =g\left(\left\|\mathbf{x}_{1}-\mathbf{x}_{2}\right\|\right) .
\end{aligned}
$$

This means that $\left\{Z(\mathbf{x}), \mathbf{x} \in \mathbb{R}^{d}\right\}$ is an intrinsically stationary random field, and all its increments are thus stationary. In particular, for a fixed location $\mathbf{x}_{0} \in \mathbb{R}^{d}$, the covariance for the increment process $\left\{Z\left(\mathbf{x}+\mathbf{x}_{0}\right)-Z(\mathbf{x}), \mathbf{x} \in \mathbb{R}^{d}\right\}$ is

$$
\begin{aligned}
& \operatorname{cov}\left(Z\left(\mathbf{x}_{1}+\mathbf{x}_{0}\right)-Z\left(\mathbf{x}_{1}\right), Z\left(\mathbf{x}_{2}+\mathbf{x}_{0}\right)-Z\left(\mathbf{x}_{2}\right)\right) \\
= & \operatorname{cov}\left(Z\left(\mathbf{x}_{1}+\mathbf{x}_{0}\right), Z\left(\mathbf{x}_{2}+\mathbf{x}_{0}\right)\right)+\operatorname{cov}\left(Z\left(\mathbf{x}_{1}\right), Z\left(\mathbf{x}_{2}\right)\right) \\
& -\operatorname{cov}\left(Z\left(\mathbf{x}_{1}+\mathbf{x}_{0}\right), Z\left(\mathbf{x}_{2}\right)\right)-\operatorname{cov}\left(Z\left(\mathbf{x}_{1}\right), Z\left(\mathbf{x}_{2}+\mathbf{x}_{0}\right)\right) \\
= & g\left(\left\|\mathbf{x}_{1}-\mathbf{x}_{2}+\mathbf{x}_{0}\right\|\right)+g\left(\left\|\mathbf{x}_{1}-\mathbf{x}_{2}-\mathbf{x}_{0}\right\|\right)-2 g\left(\left\|\mathbf{x}_{1}-\mathbf{x}_{2}\right\|\right) .
\end{aligned}
$$

In other words, the function

$$
C(\mathbf{x})=g\left(\left\|\mathbf{x}+\mathbf{x}_{0}\right\|\right)+g\left(\left\|\mathbf{x}-\mathbf{x}_{0}\right\|\right)-2 g(\|\mathbf{x}\|), \mathbf{x} \in \mathbb{R}^{d},
$$

is a covariance function on $\mathbb{R}^{d}$ for a fixed $\mathbf{x}_{0} \in \mathbb{R}^{d}$, provided that $g(\|\mathbf{x}\|)$ is a variogram. Another implication is that taking differences from an isotropic, intrinsically stationary random field achieves stationarity, but may not preserve isotropy. Whenever it exists, the directional derivative process will be stationary but not isotropic [1].

As an example of (3.2), letting $g(x)=|x|^{\alpha}, x \in[0, \infty)$, where $\alpha \in(0,2]$, yields

$$
C(\mathbf{x})=\left\|\mathbf{x}+\mathbf{x}_{0}\right\|^{\alpha}+\left\|\mathbf{x}-\mathbf{x}_{0}\right\|^{\alpha}-2\|\mathbf{x}\|^{\alpha}, \mathbf{x} \in \mathbb{R}^{d},
$$

whose univariate margin is the stationary fractional Brownian motion or the fractional Gaussian noise, which was proposed by [16] and [17] as a long-range dependent model for hydrological and geophysical time series.

We list some simple examples of $g(x), x \in[0, \infty)$ that make $g(\|\mathbf{x}\|)$ a variogram on $\mathbb{R}^{d}$ :

(i) $g(x)=(x+\beta)^{\alpha}-\beta^{\alpha}, x \geq 0$, where $\alpha \in(0,1]$ and $\beta \geq 0$;

(ii) $g(x)=x^{\alpha}\left(x^{\alpha}+1\right)^{-1}, x \geq 0$, where $\alpha \in(0,1]$;

(iii) $g(x)=1-\exp (-x), x \geq 0$;

(iv) $g(x)=\alpha^{2}-\beta^{2}-\alpha^{2} \exp (-\beta x)+\beta^{2} \exp (-\alpha x), x \geq 0$, where $0<\beta<\alpha$;

(v) $g(x)=\ln (1+x), x \geq 0$.

Each of these functions has a completely monotone derivative.

There are at least two interpretations for a bounded variogram $g(\|\mathbf{x}\|)$ on $\mathbb{R}^{d}$. As is described above, it is the variogram corresponding to an intrinsically stationary random field with the covariance of Schoenberg-Lévy type (3.1). Alternatively, $g(\|\mathbf{x}\|)$ may be thought of as the variogram associated with a stationary random field whose covariance function is of the form $\gamma_{0}-g(\|\mathbf{x}\|), \mathbf{x} \in \mathbb{R}^{d}$, where $\gamma_{0}$ is a nonnegative constant with $\gamma_{0} \geq \sup _{x \geq 0} g(x)$. Clearly, the second interpretation does not apply to an unbounded variogram $g(\|\mathbf{x}\|)$ on $\mathbb{R}^{d}$.

\section{ACKNOWLEDGEMENTS}

I am grateful to Professor Phillip E. Parker and the two referees for their helpful comments and suggestions. 


\section{REFERENCES}

1. Banerjee, S. and Gelfand, A.E., On smoothness properties of spatial processes, J. Multivariate Anal. 84 (2003), 85-100. MR1965824 (2003k:62164)

2. Berg, C., Christensen, J.P.R. and Ressel, P., Harmonic analysis on semigroups: Theory of positive definite and related functions, Springer, New York, 1984. MR0747302 (86b:43001)

3. Berg, C. and Forst, G., Potential Theory on Locally Compact Abelian Groups, Springer, New York, 1975. MR0481057 (58:1204)

4. Christakos, G., Random fields models in earth sciences, Academic Press, San Diego, 1992.

5. Christakos, G. and Papanicolaou, V., Norm-dependent covariance permissibility of weakly homogeneous spatial random fields and its consequences in spatial statistics, Stoch. Envir. Res. Risk Assess. 14 (2000), 471-478.

6. Cressie, N., Statistics for spatial data, revised ed., Wiley, New York, 1993. MR1239641 (94h:62155)

7. Doob, J.L., The elementary Gaussian processes, Ann. Math. Statist. 15 (1944), 229-282. MR0010931 (6:89a)

8. Ferguson, T.S., A representation of the symmetric bivariate Cauchy distribution, Ann. Math. Statist. 33 (1962), 1256-1266. MR0143281 (26:840)

9. Gangolli, R., Positive definite kernels on homogeneous spaces and certain stochastic processes related to Lévy's Brownian motion of several parameters, Ann. Inst. H. Poincaré B 3 (1967), 121-226. MR0215331 (35:6172)

10. Herz, C.S., A class of negative-definite functions, Proc. Amer. Math. Soc. 14 (1963), 670-676. MR0158251 (28:1477)

11. Lantuéjoul, C., Geostatistical simulation, Springer, New York, 2002.

12. Ma, C., Spatio-temporal stationary covariance models, J. Multivariate Anal. 86 (2003), 97107. MR1994723 (2004d:62329)

13. Ma, C., Spatial autoregression and related spatio-temporal models, J. Multivariate Anal. 88 (2004), 152-162. MR2021867 (2004k:62206)

14. Ma, C., Spatio-temporal variograms and covariance models, Adv. Appl. Prob. 37 (2005), 706-725. MR 2156556 (2006b:60068)

15. Ma, C., Linear combinations of space-time covariance functions and variograms, IEEE Trans. Signal Proc. 53 (2005), 857-864. MR2123904 (2005k:62173)

16. Mandelbrot, B.B., A fast fractional Gaussian noise generator, Water Resources Res. 7 (1971), 543-553.

17. Mandelbrot, B.B. and Van Ness, J.W., Fractional Brownian motions, fractional noises and applications, SIAM Rev. 10 (1968), 422-437. MR0242239(39:3572)

18. Matheron, G., The internal consistency of models in geostatistics, In Geostatistics, Vol. 1 (1989), 21-38, edited by M. Armstrong, Kluwer Academic Publishers, The Netherlands.

19. Ossiander, M. and Waymire, E.C., Certain positive-definite kernels, Proc. Amer. Math. Soc. 107 (1989), 487-492. MR.1011824 (91a:60132)

20. Sampson, P. and Guttorp, P., Nonparametric estimation of nonstationary spatial covariance structure, J. Amer. Statist. Ass. 87 (1992), 108-119.

21. Schoenberg, I.J., Metric spaces and completely monotone functions, Ann. Math. 39 (1938), 811-841. MR1503439

22. Weber, R.O. and Talkner, P., Some remarks on spatial correlation function model, Mon. Weather Rev. 121 (1993), 2611-2617. [Correction: 127 (1999), 576].

23. Yaglom, A.M., Correlation theory of stationary and related random functions, Vol. 1, Springer, New York, 1987. MR0893393 (89a:60105)

Department of Mathematics and Statistics, Wichita State University, Wichita, KANSAS 67260-0033

E-mail address: cma@math.twsu.edu 